\title{
ON THE GANEA STRONG CATEGORY IN PROPER HOMOTOPY
}

\author{
by R. AYALA and A. QUINTERO
}

(Received 23rd April 1996)

Dedicated to the memory of Dr. W. J. Rae Mitchell

\begin{abstract}
This paper contains some basic relations between Ganea strong category and Lusternik Schnirelmann category in proper homotopy theory. We focus our interest on the case of category 2 in order to show that $\mathbb{R}^{n}$ is the unique open $n$-manifold with proper Lusternik-Schnirelmann category $2(n \neq 3)$.
\end{abstract}

1991 Mathematics subject classification: 55M30.

\section{Introduction}

The Lusternik-Schnirelmann category (L-S category) $\operatorname{cat}(X)$ of a space $X$ is the smallest number $k$ such that there exists an open cover $\left\{X_{1}, \ldots X_{k}\right\}$ for which each inclusion $X_{j} \subseteq X$ is nullhomotopic in $X$. The L-S category turns to be a homotopy invariant of the space $X$. See [15] for a survey on L-S category.

Associated to the notion of L-S category is the notion of geometric category $\operatorname{gcat}(X)$ due to Fox [10], which is the smallest positive integer $k$ such that $X$ can be covered by $k$ open contractible subsets. The geometric category is not an invariant of the homotopy type of $X[10]$. The homotopy invariant version of $g c a t(X)$ was introduced by $\mathrm{T}$. Ganea in [12]. It is called strong category, $\operatorname{Cat}(X)$, and it was originally defined as the least integer $k \geq 1$ with the property that $X$ has the homotopy type of a polyhedron which may be covered by $k$ subpolyhedra each of which is contractible in itself. For a comparison of the invariants cat(-) and Cat(-) see [12] or [22].

In this paper we focus our interest on numerical invariants of the LusternikSchnirelmann type for non-compact spaces. Ordinary homotopy invariants do not describe what is happening "far out" towards infinity and more subtle invariants are needed to deal with non-compact spaces. To take the "infinity" into account one has to use proper maps instead of just continuous maps and then proper homotopy invariants replace the usual ones for non-compact spaces.

In [2] L-S category invariants are extended to the proper category $\mathcal{P}$ consisting of locally compact $\sigma$-compact Hausdorff spaces and proper maps. In this paper we define 
the proper analogue of a Ganea strong category and we relate it to a proper L-S category. As an application, we prove that any open $n$-manifold with proper $\mathrm{L}-\mathrm{S}$ category 2 is 1 -LC at $\infty$ and moreover $M$ has the proper homotopy type of $\mathbb{R}^{n}((3.13)$ and (3.15)). From this the Euclidean space $\mathbb{R}^{n}(n \neq 3)$ is characterized as the unique open $n$-manifold with proper L-S category $2((3.13))$. This result improves previous results in [2].

\section{Preliminaries}

We shall deal with the category $\mathcal{P}$ of Hausdorff locally compact $\sigma$-compact spaces and proper maps and the full subcategory $\mathcal{P}_{0} \subseteq \mathcal{P}$ consisting of connected locally pathconnected spaces in $\mathcal{P}$. Recall that a proper map ( $p$-map) is a continuous map $f: X \rightarrow Y$ such that $f^{-1}(K)$ is compact for each compact subset $K \subseteq Y$. Notice that the spaces in $\mathcal{P}$ always are paracompact $([9,7.6 .12])$.

Given a space $X$ in $\mathcal{P}$, a system of $\infty$-neighbourhoods of $X$ is a decreasing sequence $\left\{W_{j}\right\}$ of subsets of $X$ where the closures $K_{j}=\overline{X-W_{j}}$ form an increasing sequence of compact subsets with $K_{j} \subseteq$ int $K_{j+1}$ and $X=\cup i n t K_{j}$. The sequence $\left\{K_{j}\right\}$ will be called an exhausting sequence of compact subsets.

A Freudenthal end of $X$ is an element of the inverse limit $\mathcal{F}(X)=\lim \mathcal{U}\left(W_{j}\right)$, where $\mathcal{U}(-)$ stands for the family of unbounded connected components. We shall also consider the family $\mathcal{B}(-)$ of bounded components. A subset $A \subseteq X$ is termed bounded (unbounded, resp.) if the closure $\bar{A}$ is compact (non-compact, respectively). If $\mathcal{F}(X)=\{*\}$ then $X$ is said to be one-ended.

The following lemmas are commonplace in the topology of spaces in $\mathcal{P}$, but they are proved here since we do not know precise references for the proofs.

Lemma 1.1. Let $X$ be a space in $\mathcal{P}_{0}$. Given a compact connected subset $K \subseteq X$ and a locally finite sequence $\left\{A_{j}\right\}_{j \geq 1}$ of connected compact subsets of $X$ there exists a connected open neighbourhood of $K, U$, such that $\bar{U}$ is compact, and $F r U \cap A_{j}=\emptyset$ for all $j$. Moreover there also exists a compact connected neighbourhood $L$ of $K$ such that $U$, $\mathcal{B}(X-L)=\emptyset$, and $F r L \cap A_{j}=\emptyset$ for all $j$.

Proof. Since $X$ is normal, locally connected and locally compact and $\left\{A_{j}\right\}$ is locally finite we can find a finite open cover $\left\{U_{1}, \ldots, U_{m}\right\}$ of the compact connected subset $K^{\prime}=K \cup\left\{A_{j} ; A_{j} \cap K \neq \emptyset\right\}$ with $\bar{U}_{i}$ compact and $\bar{U}_{i} \subseteq X-\left\{A_{j} ; A_{j} \cap K=\emptyset\right\}$. Let $U$ be the connected component of $U\left\{U_{i} ; 1 \leq i \leq m\right\}$ containing $K^{\prime}$. Clearly $\operatorname{Fr} U \cap A_{j}=\emptyset$ and $\bar{U}$ is compact. Let $L=\bar{U} \cup\{D ; D \in \mathcal{B}(X-\bar{U})\}$. It is easy to check that $L$ is connected and $\mathcal{U}(X-L)=\mathcal{U}(X-\bar{U})$. Hence $\mathcal{B}(X-L)=\emptyset$. Moreover, $\operatorname{Fr} L \subseteq F r U$ implies $\operatorname{Fr} L \cap A_{j}=\emptyset$ for all $j$. Finally $L$ is compact. For this let $M \subseteq X$ be a compact set with $\bar{U} \subseteq$ int $M$. Then the compactness of $F r M$ yields that the family $\mathcal{A}_{1}$ of components of $X-\bar{U}$ which meet FrM is finite. Then $L-\bar{U}$ is contained in the union of the families $\mathcal{A}_{1}$ and $\mathcal{A}_{2}=\{D \in \mathcal{B}(X-\bar{U}) ; D \subseteq M\}$. As $A_{1}=\bar{\cup}\left\{D ; D \in \mathcal{A}_{1}\right\}$ and $A_{2}=\overline{\cup\left\{D ; D \in \mathcal{A}_{2}\right\}} \subseteq M$ are both compact sets it follows that $L \subseteq \bar{U} \cup A_{1} \cup A_{2}$ is also compact. 
Lemma 1.2. Let $X$ be $a$ space in $\mathcal{P}_{0}$. Then there exists a sequence $U_{1} \subseteq L_{1} \subseteq U_{2} \subseteq L_{2} \ldots$ such that $U_{i}$ is a connected open set and $L_{i}$ is compact with $\mathcal{B}\left(X-L_{i}\right)=\emptyset$. Moreover, if $\left\{A_{j}\right\}_{j \geq 1}$ is a locally finite sequence of compact connected subsets of $X$ the $U_{i}$ 's and $L_{i}$ 's can be chosen in such a way that each $A_{j}$ is contained in $a$ difference $U_{i}-L_{i-1}(i \geq 1)$ with $L_{0}=\emptyset$.

Proof. Let $K_{1} \subseteq K_{2} \ldots$ be an exhausting sequence of compact subsets. Since $K_{1} \subseteq$ int $K_{2}$ we can find a finite open cover $\left\{W_{k}\right\}$ with $W_{k} \subseteq$ int $K_{2}$ connected and $\bar{W}_{k}$ compact. Moreover, we can join the connected components of $W=U\left\{W_{k}\right\}$ by finite chains of connected open subsets of compact closures in some int $K_{i_{1}}$. Let $V_{1}$ denote the union of all the open subsets which form these chains. Then $V_{1}$ is connected and open and $\bar{V}_{1} \subseteq \operatorname{int} K_{i_{1}}$ is compact. Hence the union $H_{1}=\bar{V}_{1} \cup\left\{A_{j} ; A_{j} \cap \bar{V}_{1} \neq \emptyset\right\}$ is compact and connected and contained in some int $K_{j_{1}}$ with $j_{1} \geq i_{1}$. Since $X$ is normal and $\left\{A_{j}\right\}$ is locally finite we can find an open set $Z_{1}$ with $H_{1} \subseteq Z_{1} \subseteq \bar{Z}_{1} \subseteq$ int $K_{j_{1}}-\cup\left\{A_{j} ; A_{j} \cap \bar{V}_{1}=\emptyset\right\}$. Let $U_{1}^{\prime}$ denote the connected component of $Z_{1}$ which contains $H_{1}$. Now we apply (1.1) to $\overline{U_{1}^{\prime}}$ and we get an open connected subset $U_{1}$ and a compact connected set $L_{1}$ with $\overline{U_{1}^{\prime}} \subseteq U_{1} \subseteq L_{1}$ and $\mathcal{B}\left(X-L_{1}\right)=\emptyset$ and moreover $A_{j} \cap F r U_{1}=\emptyset$ for all $j$. Assume $L_{1} \subseteq \operatorname{int} K_{s_{1}}$. If we replace $K_{1}$ and $K_{2}$ by $L_{1}$ and $K_{s_{1}}$ respectively, an inductive process can now be started to construct the required sequence.

The usual notions of homotopy, deformation, homotopy equivalence, etc . . . can be defined in the natural way in $\mathcal{P}$. Actually, the ordinary cylinder functor $I X=X \times I$ endows the category $\mathcal{P}$ of a structure of $I$-category and hence $\mathcal{P}$ is a cofibration category in the sense of Baues (see [5] and [1]). The symbols $\simeq, \simeq_{p}$, and $\cong$ stand for homotopy equivalence, p-homotopy equivalence, and homeomorphism respectively. The set of p-homotopy classes of p-maps $f: X \rightarrow Y$ will be denoted by $[X, Y]_{p}$. A ray is a proper map $\alpha: J \rightarrow X$ where $J$ denotes the half-line $J=[0, \infty)$. A strong end of $X$ is the p-homotopy class of a ray in $F(X)=[J, X]_{p}$. Clearly each strong end defines a Freudenthal end. When $F(X)=\{*\}$ we say that $X$ is strongly one-ended.

In the category $\mathcal{P}$ the constant map $X \rightarrow\{*\}$ is not defined if $X$ is not compact. However the role of the point is played in $\mathcal{P}$ by the half-line $J$. For this we recall that for any space $X$ in $\mathcal{P}$, there always exists a proper map $r: X \rightarrow J([8 ; 6.3 .5])$. Moreover the map $r$ is unique up to p-homotopy. We shall briefly describe the construction of such a map $r$. If $\left\{U_{j}\right\}_{j \geq 0}$ is a system of $\infty$-neighbourhoods of $\boldsymbol{X}$ with $U_{0}=X$, the Tietze Extension Theorem yields continuous maps $r_{j}=\overline{U_{j}-U_{j+1}} \rightarrow[j, j+1]$ with $r_{j}\left(F r U_{j+1}\right)=j+1, r_{j}\left(F r U_{j}\right)=j$. It is now clear that the maps $r_{j}$ define a proper map $r: J \rightarrow X$. By using $r$, we can define (up to p-homotopy) the proper cone $C_{p} X$ as the push-out in $\mathcal{P}$ of the diagram $X \times I \stackrel{i_{0}}{\leftarrow} X \stackrel{r}{\rightarrow} J$. Also if $f: X \rightarrow Y$ is a proper map, the proper cone of $f, C_{p} f$ is naturally defined in $\mathcal{P}$. When $f=r$, the proper cone $C_{p} r=\Sigma_{p} X$ is called the proper suspension. Since $J$ is contractible it is not hard to check that the proper homotopical constructions in the $I$-category $\mathcal{P}$ have the same ordinary homotopy type as the ordinary ones.

The following lemma leads us to the proper analogue of Borsuk's homotopy extension theorem $([19$, p. 41$])$. Recall that a space $X$ is said to be an absolute 
neighbourhood retract (ANR-space) if for any metrizable space $Y$ and any continuous map $f: A \rightarrow X$ with $A \subseteq Y$ closed $f$ admits a continuous extension $\tilde{f}: U \rightarrow X$ for some neighbourhood $U$ of $A$.

Lemma $1.3([4,3.5])$. Let $X$ be a locally compact metrizable space and $Y$ a locally compact $A N R$ in $\mathcal{P}$. Let $C \subseteq X$ be a closed subset and $f, g: C \rightarrow Y$ p-homotopic maps. If $W \subseteq X$ is open with $C \subseteq W$ and $\tilde{f}, \tilde{g}: W \rightarrow Y$ are continuous extensions of $f$ and $g$ respectively then there exists a closed neighbourhood $U$ of $C$ in $W$ such that $\left.\tilde{f}\right|_{U}$ and $\left.\tilde{g}\right|_{U}$ are p-homotopic.

Proposition 1.4. Every $A N R$ in $\mathcal{P}$ has the proper homotopy extension property $(P E H P)$ with respect to all pairs $(X, A)$ in $\mathcal{P}$ with $A$ closed and $X$ metrizable.

Proof. It is similar to the classical proof in $([19$, p. 41$])$. If $V$ is any closed neighbourhood of $A$ in $X$ and $C=X \times\{0\} \cup A \times I$, let $\phi: X \rightarrow I$ be a Urysohn map with $\phi(A)=1$ and $\phi(X-$ int $V)=\emptyset$. Then $r: X \times I \rightarrow X \times I$ given by $r(x, y)=(x, \phi(x) t)$ is a p-map such that $\left.r\right|_{C}=i d_{c}$ and $r(X \times I) \subseteq V \times I$.

Now let $H: C \rightarrow Y$ a p-map with $Y$ an ANR in $P$. Since $Y$ is an ANR we can extend $H$ to a continuous map $\tilde{H}: W \rightarrow Y$ where $\mathrm{W}$ is an open neighbourhood of $C$ in $X \times I$. Then there exists a closed neighbourhood $U \subseteq W$ of $C$ such that $\left.\tilde{H}\right|_{C}$ is proper. Here we use (1.3) with $f=g=H$. By using the compactness of $I$ we can find a closed neighbourhood $V$ of $A$ such that $V \times I \subseteq U$. Then $\tilde{H} r: X \times I \rightarrow Y$ is a proper extension of $H$.

We also derive from (1.3) the following lemma which will be used later.

Lemma 1.5. Let $X$ be a metrizable ANR-space in $\mathcal{P}$ and let $C$ be closed in $X$. Assume that the inclusion $i_{c}: C \subseteq X$ is p-homotopic to a composition of p-maps $C \stackrel{\leftrightarrow}{\rightarrow} J \stackrel{\leftrightarrow}{\rightarrow} X$. Then there exists a closed neighbourhood $U$ of $C$ and a proper extension $r^{\prime}: U \rightarrow J$ such that the composition $\alpha r^{\prime}$ is $p$-homotopic to the inclusion $U \subseteq X$.

Proof. Let $r_{0}: X \rightarrow J$ be any continuous extension of $r$. Here we use the Tietze Extension Theorem. If $H: i_{c} \simeq_{p} \alpha r$ is a p-homotopy we consider the map

$$
h=i d_{X} \cup H \cup \alpha r_{0}: X \times\{0,1\} \cup C \times I \rightarrow X .
$$

As $X$ is an ANR-space we can extend $h$ to $\tilde{h}: W \rightarrow X$ for some neighbourhood $W$ of $X \times\{0,1\} \cup C \times I$ in $X \times I$. By using the compactness of $I$ one finds a neighbourhood $V$ of $C$ in $X$ with $V \times I \subseteq W$. Let $r_{1}=r_{0} \mid V$. Then $\tilde{h} \mid V \times I$ yields a homotopy $i_{v} \simeq \alpha r_{1}$. As an immediate consequence of (1.3) we get a closed neighbourhood $U \subseteq V$ and a phomotopy $i_{U} \simeq_{p} \alpha r^{\prime}$ where $r^{\prime}=r_{1} \mid U$. 


\section{Numerical proper homotopy invariants}

In [2] the proper L-S category of a space in $\mathcal{P}$ was defined. Namely, a closed subset $A \subseteq X$ is said to be properly deformable to $J$ in $X$ if there exists a diagram in $\mathcal{P}$

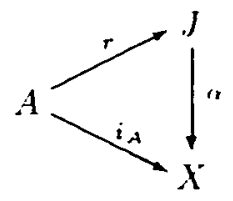

which is commutative up to p-homotopy. That is, there exists a p-homotopy $H: i_{A} \simeq_{p} \alpha r$. Notice that we can use the restriction to $A$ of any proper map $r: X \rightarrow J$ but the p-homotopy class of $\alpha: J \rightarrow X$ can depend on $A$. An open cover of $X\left\{V_{i}\right\}$ is said to be properly categorical (p-categorical) if each closure $\vec{V}_{i}$ is p-deformable to $J$ in $X$. The proper Lusternik Schnirelmann category (p-category) of $X, p-\operatorname{cat}(X)$, is the least number $n$ such that $X$ admits a p-categorical open cover with $n$ elements. It is not hard to check that $p-\operatorname{cat}(-)$ is a proper homotopy invariant. Moreover, the inequality $p-\operatorname{cat}(X) \geq \max \{\operatorname{Card}(\mathcal{F}(X)), \operatorname{cat}(X)\}$ always holds. Here cat denotes the ordinary L-S category. Also it is clear that $\mathbb{R}^{n}$ verifies that $p-\operatorname{cat}\left(\mathbb{R}^{n}\right)=2$. As in ordinary homotopy closed covers can be used in the definition of proper L-S category for ANR in $\mathcal{P}$. This is an immediate consequence of (1.5).

The proper analogue of Borsuk's homotopy extension theorem (1.4) yields

Proposition 2.1. Let $X$ be a metrizable $A N R$ in $\mathcal{P}$ and let $P \subseteq X$ be a closed subspace p-deformable to $J$ in $X$. If $\left\{C_{i}\right\}_{i \geq 1}$ is a locally finite sequence of pairwise disjoint compact subspaces of $P$ and $x_{i} \in C_{i}$ there exists a $p$-deformation of $\cup_{i \geq 1} C_{i}$ to $A=\left\{x_{i}\right\}_{i \geq 1}$ relative to $A$. Moreover, this p-deformation can be extended to a p-deformation of $P$ to $J$ in $X$.

Proof. The result is a consequence of the proper homotopy extension property (PHEP) in (1.4). We shall give some details. Assume we have a diagram

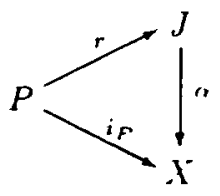

and a p-homotopy $H: i_{P} \simeq_{p} \alpha r$. Since any two p-maps in $J$ are p-homotopic we can use the PHEP of $\left(P, \cup C_{i}\right)$ to change $r$ for a new $r_{1}$ with $r_{1}\left(C_{i}\right)=i \in \mathbb{N}$ for all $i \geq 1$. We have a new p-homotopy $H^{1}: i_{P} \simeq_{p} \alpha r_{1}$. By using the PHEP of $(J, \mathbb{N})$ we replace $\alpha$ by $\alpha_{1} \simeq_{p} \alpha$ with $\alpha_{1}(i)=x_{i}$. Here we use the paths $\left.H^{1}\right|_{\left\{x_{i}\right\} \times I}$ between $\alpha(i)$ and $x_{i}$ given by the p-homotopy $H^{1}$. Let $H^{2}: i_{P} \simeq_{p} \alpha_{1} r_{1}$ be a p-homotopy. 
By using again the (PEHP) of $(J, \mathbb{N})$ we find $\alpha_{2}$ and a p-homotopy $F: \alpha_{1} \simeq \alpha_{2}$ such that $F(i, t)=H^{2}\left(x_{i}, 1-t\right)$ for all $i \in \mathbb{N}$. Then by putting together the homotopies $H^{2}$ and $F(r \times i d)$ we get a new p-homotopy $H^{3}: i_{p} \simeq \alpha_{2} r_{1}$ such that $H^{3} \mid\left\{x_{i}\right\} \times I$ is a loop $\gamma_{i}$ homotopic to the constant path $c_{x_{i}}$. Moreover the homotopy $G_{i}: \gamma_{i} \simeq c_{x_{i}}$ rel $\left\{x_{i}\right\} \times\{0,1\}$ can be chosen with $G_{i}\left(\left\{x_{i}\right\} \times I \times I\right) \subseteq H^{2}\left(\left\{x_{i}\right\} \times I\right)$ and so we have a p-homotopy $G=\cup G_{i}: A \times I \times I \rightarrow X$ rel $A \times\{0,1\}$. Finally we apply the (PEHP) to the pair.

$$
(P \times I \times I, P \times I \times\{0\} \cup P \times\{0,1\} \times I \cup A \times I \times I)
$$

to extend $H^{3} \cup i_{P} \times\{0\} \times I \cup \alpha_{2} r_{1} \times\{1\} \times I \cup G$ to a p-homotopy $H^{4}: P \times I \times I \rightarrow X$. Then $H^{4} \mid P \times I \times\{1\}: i_{P} \simeq \alpha_{2} r_{1}$ is the required p-deformation of $P$ extending a $\mathrm{p}$ deformation of $C$ onto $A$ relatively to $A$.

A subspace $A \subseteq X$ in $\mathcal{P}$ will be called properly admissible if the family of connected components of $A$ is locally finite in $X$ (and hence countable).

Lemma 2.2. Let $\left\{U_{1}, \ldots, U_{n}\right\}$ be an open p-categorical cover of a space $X$ in $\mathcal{P}_{0}$. Then there exists an open p-categorical cover $\left\{U_{1}^{\prime}, \ldots, U_{n}^{\prime}\right\}$ such that each $\overline{U_{i}^{\prime}}$ is properly admissible.

Proof. Let $K_{1} \subseteq K_{2} \ldots$ be an exhausting sequence of compact subsets of $X$. Let $\mathcal{C}_{m}\left(U_{i}\right)$ denote the family of path components of $U_{i}-K_{m}$. Here $m \geq 0$ with $K_{0}=\emptyset$. Since $X$ is locally path connected each $C \in \mathcal{C}_{m}\left(U_{i}\right)$ is an open subset. By compactness there exist finite families $\mathcal{C}_{m}^{\prime}\left(U_{i}\right) \subseteq \mathcal{C}_{m}\left(U_{i}\right)$ such that $\overline{K_{m+1}-K_{m}} \subseteq \cup\left\{C ; C \in \mathcal{C}_{m-1}^{\prime}\left(U_{i}\right)\right\}(m \geq 0)$. Here $\mathcal{C}_{-1}^{\prime}\left(U_{i}\right)=\mathcal{C}_{0}\left(U_{i}\right)=\mathcal{C}\left(U_{i}\right)$. For each $i$ we define $U_{i}^{\prime}=\cup\left\{C ; C \in \mathcal{C}_{m}^{\prime}\left(U_{i}\right) ; m \geq 0\right\}$. It is clear that $\left\{U_{i}^{\prime}\right\}_{1 \leq i \leq n}$ is an open cover and it is p-categorical since $U_{i}^{\prime} \subseteq U_{i}$. Moreover the family $\mathcal{C}\left(U_{i}^{\prime}\right)$ of path components of $U_{i}^{\prime}$ is locally finite since any point in $K_{m}$ only meets path components of $\mathcal{C}_{j}^{\prime}\left(U_{i}\right)$ with $j \leq m-1$. Furthermore, if $D \subseteq \overline{U_{i}^{\prime}}$ is a connected component then $D=\cup\left\{\bar{C} ; C \in \mathcal{C}\left(U_{i}^{\prime}\right)\right.$ and $\left.C \subseteq D\right\}$. Indeed, since $D$ is closed $D$ contains the union of closures. Conversely, if $x \in D$ we have that $x \in \bar{C}$ with $C \in \mathcal{C}\left(U_{i}^{\prime}\right)$ since $\mathcal{C}\left(U_{i}^{\prime}\right)$ is locally finite. As $\bar{C} \subseteq \overline{U_{i}^{\prime}}$ is connected it follows $\bar{C} \subseteq D$. Finally the family of connected components of $\overline{U_{i}^{\prime}}$ is locally finite since given an open $G$ which only meets the path components $C_{1} \ldots C_{k}$ of $U_{i}^{\prime}$ then $G$ only meets the components $D \subseteq \overline{U_{i}^{\prime}}$ which contain some $C_{s}(1 \leq s \leq k)$.

Proposition 2.3. Assume $X$ is a one-ended metrizable ANR-space in $\mathcal{P}_{0}$ and $H_{1}\left(X ; \mathbb{Z}_{2}\right)=0$. If $P$ is a properly admissible closed subset p-deformable to $J$ with $\mathcal{U}(P) \neq \emptyset$ there exists a closed subset $P^{\prime} \subseteq X$ with $P \subseteq P^{\prime}, \mathcal{B}\left(P^{\prime}\right)=\emptyset$, and such that $P^{\prime}$ is $p$-deformable to $J$ in $X$.

Proof. Let $\emptyset=L_{0} \subseteq U_{1} \subseteq L_{1} \ldots$ be an increasing sequence with the properties of (1.2). Moreover we can assume that each bounded component $B \in \mathcal{B}(P)$ is contained in some difference $D_{k}=U_{k}-L_{k-1}(k \geq 1)$. We shall also use the differences $D_{k}^{j}=U_{k}-L_{j-1}$ for $j \leq k$. Note $D_{k}^{k}=D_{k}$. Since both $X-L_{j}$ and $U_{k}$ are open connected 
and hence path connected sets, the reduced Mayer-Vietoris exact sequence yields the isomorphism

$$
0=H_{1}\left(X ; \mathbb{Z}_{2}\right) \rightarrow \tilde{H}_{0}\left(D_{k}^{j} ; \mathbb{Z}_{2}\right) \rightarrow \tilde{H}_{0}\left(X-L_{j-1} ; \mathbb{Z}_{2}\right) \oplus \tilde{H}_{0}\left(U_{k} ; \mathbb{Z}_{2}\right) \rightarrow \tilde{H}_{0}\left(X ; \mathbb{Z}_{2}\right)=0
$$

and so each $D_{k}^{j}$ is path connected.

In order to connect all the bounded components of $P$ to unbounded components we now proceed in each difference $D_{k}$ in the same way as in $([10,16.2])$. Namely, we construct for each $k \geq 1$ a union ("forest") $F_{k}=\cup T_{j}^{k}$ of pairwise disjoint directed "trees" $T_{j}^{k} \subseteq D_{k}$ whose "vertices" are all bounded components $B \in \mathcal{B}(P)$ with $B \subseteq D_{k}$ together with some points in $D_{k}$. Furthermore we shall construct each $F_{k}$ in such a way that the source vertices are bounded components and the sink vertices are points of unbounded components which meet $D_{k}$. Hence for each bounded component $B \subseteq T_{j}^{k}$ there will be only one directed path $\gamma_{B}$ from a point $x_{B} \in B$ to some unbounded component $U_{B} \in \mathcal{U}(P)$. The forest $F_{k}$ is constructed inductively as follows. Assume we have already constructed a forest $F_{k}^{\prime}$. Let $m_{k}^{\prime}$ denote the number of bounded components in $D_{k}-F_{k}^{\prime}$. Then we consider an arc $\gamma$ from a bounded component $B \subseteq D_{k}-F_{k}^{\prime}$ to some unbounded component $U$ with $U \cap D_{k} \neq \emptyset$. Let $p$ be the first point in $\gamma \cap\left(F_{k}^{\prime} \cap U\right)$ and let $q$ be the last point in the subarc from $B$ to $p$ belonging to some component $B^{\prime} \subseteq D_{k}-F_{k}^{\prime}$. Let $\gamma^{\prime} \subseteq \gamma$ be the subarc between $q$ and $p$, and let $F_{k}^{\prime \prime}=F_{k}^{\prime} \cup \gamma^{\prime} \cup B^{\prime}$. Then $m_{k}^{\prime \prime}=m_{k}^{\prime}-1$. Hence any forest $F_{k}^{\prime}$ can be extended to a forest $F_{k}$ with $m_{k}=0$. The result follows by starting with $F_{k}^{\prime}=\emptyset$.

Once we have constructed a complete forest $F_{k}$ in each $D_{k}$, we follow the above argument to extend $F_{k} \cup F_{k-1}$ to a new forest $F_{k}^{k-1}$ in the difference $D_{k}^{k-1}$ containing all components in $D_{k}^{k-1}$. Then $F=\cup\left\{F_{k}^{k-1} ; k \geq 2\right\}$ is a locally finite forest in $X$ containing all components in $\mathcal{B}(P)$. Now we use $(2.1)$ to choose a p-deformation of $P$ relatively to each point $x_{B}, B \in \mathcal{B}(P)$. The construction of the (directed) forest yields an order to push each tree $T \subseteq F$ onto $\Omega_{P}=\cup\{U ; U \in \mathcal{U}(P)\}$. Note that this deformation is proper since any $x_{B}$ is pushed along a finite tree inside $D_{k}^{k-1}$ if $B \subseteq D_{k}^{k-1}$. Hence $P^{\prime}=P \cup F$ is p-deformable onto $P$ in $X$ and so $P^{\prime}$ is p-deformable to $J$ in $X$. This finishes the proof.

Proposition 2.4. Let $X$ be a one-ended $A N R$-space in $\mathcal{P}_{0}$ with $H_{1}\left(X ; \mathbb{Z}_{2}\right)=0$. Assume that $\{P, Q\}$ is a properly admissible p-categorical cover of $X$. Then there exists a new closed cover $\left\{P^{\prime}, Q^{\prime}\right\}$ with the same properties and such that the intersection $P^{\prime} \cap Q^{\prime}$ also is properly admissible. Moreover, if $\mathcal{B}(P)=\mathcal{B}(Q)=\emptyset$ then $\mathcal{B}\left(P^{\prime} \cap Q^{\prime}\right)=\emptyset$.

Proof. Let $\left\{C_{m}\right\}$ be the connected components of $P$. If $\left\{K_{i}\right\}$ is an exhausting sequence of compact subsets of $X$ there exists an increasing sequence of positive integers $m_{1}<m_{2} \ldots$ such that for each $i \geq 1 C_{m} \cap K_{i}=\emptyset$ if $m \geq m_{i}$ since $\left\{C_{m}\right\}$ is locally finite. As $X$ is normal and locally connected we can inductively construct connected open subsets $U_{m}$ with $C_{m} \subseteq U_{m} \subseteq \bar{U}_{m} \subseteq X-\left(\cup_{j<m} \bar{U}_{j}\right) \cup\left(\cup_{k>m} C_{k}\right), U_{m} \cap U_{m^{\prime}}=\emptyset$ if $m \neq m^{\prime}$ and $U_{m} \subseteq X-K_{i}$ if $m \geq m_{i}$. Hence $\left\{\bar{U}_{m}\right\}$ is a locally finite family of pairwise disjoint closed 
sets and we define $P^{\prime}=\cup\left\{\bar{U}_{m}\right\}$. Notice that $P^{\prime}$ can be assumed p-deformable to $J$ in $X$ by (1.5), and $\mathcal{B}\left(P^{\prime}\right)=\emptyset$ if $\mathcal{B}(P)=\emptyset$ since the components of $P^{\prime}$ are $\bar{U}_{m}$. Similarly we can construct $Q^{\prime}=\cup\left\{\bar{W}_{n}\right\}$ with $W_{n}$ connected and open. On the other hand, since $H_{1}\left(X ; \mathbb{Z}_{2}\right)=0$ the Mayer-Vietoris exact sequence yields the short exact sequence

$$
0=H_{1}\left(X ; \mathbb{Z}_{2}\right) \stackrel{\Delta}{\rightarrow} H_{0}\left(U \cap W ; \mathbb{Z}_{2}\right) \rightarrow H_{0}\left(U ; \mathbb{Z}_{2}\right) \oplus H_{0}\left(W ; \mathbb{Z}_{2}\right) \rightarrow H_{0}\left(X ; \mathbb{Z}_{2}\right)=\mathbb{Z}_{2} \rightarrow 0
$$

where $U=\cup\left\{U_{m}\right\}$ and $W=\cup\left\{W_{n}\right\}$. This implies that the (path) components of $U \cap W$ are of the form $U_{m} \cap W_{n}$. Moreover we next show that the components of $P^{\prime} \cap Q^{\prime}$ are of the form $\overline{U_{m} \cap W_{n}}$. Indeed, let $C \subseteq P^{\prime} \cap Q^{\prime}$ be a component. Then there exist $m$ and $n$ such that $C \subseteq \bar{U}_{m} \cap \bar{W}_{n}$. But $\bar{U}_{m} \cap \bar{W}_{n}=\bar{U}_{m} \cap \bar{W}_{n}$ since any $x \in \bar{U}_{m} \cap \bar{W}_{m}$ with $x \notin U_{m}$ necessarily belongs to $W_{n}$ because $X=U \cup W$. Hence, for any open neighbourhood $\Omega$ of $x, \Omega \cap W_{n} \cap U_{m} \neq \emptyset$ and so $x \in \bar{U}_{m} \cap W_{n}$. Similarly if $x \notin W_{n}$. As $U_{m} \cap W_{n}$ is a (path) component of $U \cap W$ then $\overline{U_{m} \cap W_{n}} \subseteq P^{\prime} \cap Q^{\prime}$ is connected and hence $C=\overline{U_{n} \cap W_{m}}$.

Finally, if $\mathcal{B}(P)=\mathcal{B}(Q)=\emptyset$ the $U_{n}$ and $W_{n}$ are unbounded for all $m, n$. Assume that for some intersection $U_{m} \cap W_{n}$ is bounded. Then there exists a compact subset $K \subseteq X$ with $U_{m} \cap W_{n} \subseteq K$. As $X$ is one-ended we can find another compact subset $L$ with $K \subseteq L$ and such that any two points in $X-L$ can be joined by an arc in $X-K$. Since $U_{m}$ and $W_{n}$ are unbounded we can find points $x \in U_{m}-L$ and $y \in W_{n}-L$. Let $\gamma \subseteq X-K$ be a path joining $x$ to $y$. In addition paths $\alpha \subseteq U_{m}$ and $\beta \subseteq W_{n}$ can be found joining $x_{0} \in U_{m} \cap W_{n}$ to $x$ and $y$ respectively. In this way we have constructed a loop $\xi=\gamma \cup \alpha \cup \beta$ which represents an element $w_{\xi} \in H_{1}\left(X ; \mathbb{Z}_{2}\right)=0$, and its image $\Delta\left(w_{\xi}\right) \in H_{0}\left(U \cap W ; \mathbb{Z}_{2}\right)$ is the sum of components of $U \cap W$ which are crossed by $\xi$ when $\xi$ passed from $U$ to $W$. As $\gamma$ joins $U_{m}$ to $W_{n}$ outside $U_{m} \cap W_{n}$, it follows that $\Delta\left(w_{\xi}\right) \neq 0$ which is a contradiction. Therefore any intersection $U_{m} \cap W_{n}$ is unbounded and hence any component $C \subseteq P^{\prime} \cap Q^{\prime}$ is unbounded.

In addition to the L-S category, another numerical homotopy invariant was introduced by $T$. Ganea ([12]) with the name of strong category. This notation admits the following generalization to proper homotopy. Given a space $X$ in $\mathcal{P}$, the proper strong category of $X$ is the smallest number $p-\operatorname{Cat}(X)=k$ such that there exists a space $Y$ in $\mathcal{P}$ with $X \simeq_{p} Y$ and such that $Y$ is covered with the interiors of $k$ closed subspaces $Y_{i} \simeq_{p} J(1 \leq i \leq k)$. Many results concerning strong category have their corresponding analogues in proper homotopy. We shall prove below two of them (cf. $([6,5.4])$ and $([6,5.5])$.$) which will be used later on. The key point in the proofs is the$ fact that $\mathcal{P}$ is an $I$-category in the sense of [5]. In particular, categorical wedge and cone constructions are defined in $\mathcal{P}$ up to p-homotopy equivalence. Moreover, if $i: A \nrightarrow X$ is a p-cofibration in $\mathcal{P}$, the cofibre of $i$ is defined up to p-homotopy by the push-out diagram

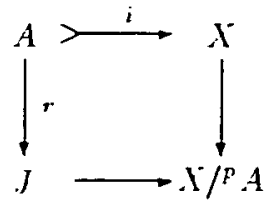


It can be shown that $X /^{p} A$ is p-homotopically equivalent to the proper mapping cone of $i C_{p} i$. In general any space $X$ p-homotopically equivalent to $X /^{p} A$ will be called the homotopy cofibre of $i$.

Proposition 2.5. Let $X$ be a space in $\mathcal{P}$ such that $p-\operatorname{cat}(X) \leq n$. Then $X$ is a p-retract of a space $\tilde{X}$ in $\mathcal{P}$ with $p-\operatorname{Cat}(\tilde{X}) \leq n$ and conversely. in $\mathcal{P}$

Proof. Let $\left\{W_{k}\right\}_{1 \leq k \leq n}$ be a p-categorical open cover of $X$. Then we have diagrams

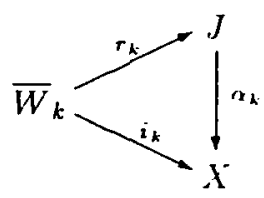

which are commutative up to p-homotopies $H^{k}$. Let $C_{p} \bar{W}_{k}$ denote the proper cone constructed by using $r_{k}$. The homotopies $H^{k}$ and the push-out properties of $C_{p} \bar{W}_{k}$ yield p-maps $\tilde{H}^{k}: C_{p} \bar{W}_{k} \rightarrow X$ such that $\tilde{H}^{k} \mid \bar{W}_{k}$ is the inclusion $i_{k}$. Hence the union of the pmaps $\tilde{H}^{k}$ give a p-retract $r: \tilde{X}=\cup\left\{C_{p} \bar{W}_{k}\right\} \rightarrow X$. Clearly each cone $C_{p} \bar{W}_{k}$ is phomotopically trivial, and so $p-\operatorname{Cat}(\tilde{X}) \leq n$. The converse is easy, since the existence of a p-retraction as above implics $p-\operatorname{cat}(X) \leq p-\operatorname{Cat}(\tilde{X})$.

Proposition 2.6. If $X$ is a space in $\mathcal{P}$ then $p-\operatorname{Cat}(X) \leq n+k$ if and only if $X$ has the p-homotopy type of the push-out, $Z_{A}\left(X_{0}, X_{1}\right)$, of a diagram $X_{0} \cup X_{1} \leftarrow A \cup A \stackrel{\left(i_{0}, i_{1}\right)}{\longrightarrow} A \times I$ (double mapping cylinder) where $p-\operatorname{Cat}\left(X_{0}\right) \leq n$ and $p-\operatorname{cat}\left(X_{1}\right) \leq k$.

Proof. The sufficiency is clear. Let now $Y \simeq_{p} X$ with $Y=\cup_{1 \leq i \leq n+k} i n t Y_{i}, Y_{i}$ closed and $Y_{i} \simeq_{p} J$. Then $X_{0}=\cup_{1 \leq i \leq n} Y_{i}=\cup_{1 \leq i \leq n}$ int $_{X_{0}} Y_{i}$ and $X_{1}=\cup_{n+1 \leq i \leq n+k} Y_{i}=\cup_{n+1 \leq i \leq n+k} i n t_{X_{1}} Y_{i}$ verify $p-\operatorname{Cat}\left(X_{0}\right) \leq n$ and $p-\operatorname{Cat}\left(X_{1}\right) \leq k$. Moreover, we claim that $Y$ has the same p-homotopy type as the double mapping cylinder $Z_{A}\left(X_{0}, X_{1}\right)$ where $A=X_{0} \cap X_{1}$. Indeed, we consider the natural closed embedding $Z_{A}\left(X_{0}, X_{1}\right) \subseteq Y \times I$ with $X_{0} \subseteq Y \times\{0\}$ and $X_{1} \subseteq Y \times\{1\}$. As $Y$ is paracompact let $\left\{\phi_{i}\right\}$ be a partition of unity subordinate to the open cover $\left\{\right.$ int $\left.Y_{i}\right\}$. We now define the p-map $\psi: Y \rightarrow Y \times I$ by $\psi(y)=\left(y, \phi_{X_{1}}(y)\right)$ where $\phi_{X_{1}}=\Sigma\left\{\phi_{i} ; i \geq n+1\right\}$. Clearly, $\phi_{X_{1}}(y)=0$ and $\phi_{X_{1}}(y)=1$ if $y \notin X_{1}$ and $y \notin X_{0}$ respectively. Therefore $\psi(Y) \subseteq Z_{A}\left(X_{0}, X_{1}\right)$.

Let $\psi^{\prime}: Y \rightarrow Z_{A}\left(X_{0}, X_{1}\right)$ and $q: Z_{A}\left(X_{0}, X_{1}\right) \rightarrow Y$ denote the restrictions of $\psi$ and the projection $\pi_{1}: Y \times I \rightarrow Y$ respectively. Clearly $q \psi^{\prime}=i d_{Y}$. Moreover the formula $H(w, s)=\left(\pi_{1}(w),(1-s) \phi_{X_{1}}^{\prime}(w)+s \pi_{2}(w)\right)$ where $\phi_{X_{1}}^{\prime}(w)=\phi_{X_{1}} \pi_{1}(w)$ yields a well-defined p-map which gives us a p-homotopy $H: \psi^{\prime} q \simeq_{p} i d_{z_{1}\left(x_{0}, x_{1}\right)}$.

Corollary 2.7. (cf. $[12,2.1])$ ) If $X$ is a connected space in $\mathcal{P} p=\operatorname{Cat}(X) \leq n$ if and only if there exists a cofibration $i: A \mapsto Y$ in $\mathcal{P}$ such that $p-\operatorname{Cat}(Y) \leq n-1$ and $X$ is the homotopy cofibre of $i$. 
Proof. According to (2.6) we have $X \simeq_{p} Z_{A}\left(X_{0}, X_{1}\right)$ for a certain double mapping cylinder with $X_{0} \simeq_{p} J$ and $p-\operatorname{Cat}\left(X_{1}\right) \leq n-1$. By general properties of cofibration categories $([5, I .1 .1])$ the natural p-map $X_{0} \mapsto Z_{A}\left(X_{0}, X_{1}\right)$ is a cofibration in $\mathcal{P}$ and the p-homotopy equivalence $X_{0} \simeq_{p} J$ yields a p-homotopy equivalence $Z_{A}\left(X_{0}, X_{1}\right) \simeq_{p} C=$ $Z_{A}\left(X_{0}, X_{1}\right) /^{p} X_{0}$. As $X$ is connected we have $A \neq \emptyset$ and clearly $C$ is homeomorphic to the mapping cone of $i: A \hookrightarrow X_{1}$ which is the push-out of the diagram $J \leftarrow A \longmapsto M_{i}$ where $M_{i}$ denotes the mapping cylinder of $i$ in $\mathcal{P}$. As $M_{i} \simeq_{p} X_{1}, p-\operatorname{Cat}\left(M_{i}\right) \leq n-1$ and the result follows.

Remark 2.8. Corollary (2.7) can be restated in the following way. The space $X$ verifies $p-\operatorname{Cat}(X) \leq n$ if and only if there exist cofibration sequences $A_{i} \mapsto X_{i} \rightarrow X_{i+1}$ $(1 \leq i \leq n-1)$ in $\mathcal{P}$ where $X_{1} \simeq_{p} J$ and $X_{n} \simeq_{p} X$. Similarly, the Cornea cone-length invariant ([7]) can also be defined in $\mathcal{P}$. Namely, the proper cone-length of $X$ is $\leq n$ if there exist cofibration sequences as above where $A_{i}=\Sigma_{p}^{i} Z_{i}$ for some space $Z_{i}$ in $\mathcal{P}$. These definitions suggest natural abstract notions of Ganea strong category and Cornea cone-length in cofibration categories. The abstract notions of both L-S category and Cornea cone-length have been already developed for J-categories in [14] and [7] respectively. Since the proper category $\mathcal{P}$ provides an example of cofibration category which does not have a natural structure of $\mathrm{J}$-category, it seems to be interesting to develop the basic properties of abstract numerical homotopy invariants in the setting of cofibration categories.

Incidentally the proper analogue of the Takens bound Cat $\leq$ cat +1 ([24]) still holds for strongly one-ended spaces (cf. [6]). In fact, the short proof of this result due to Ganea $([24, \S 5])$ is based on results available in any cofibration category. Hence one can prove

Proposition 2.9. If $X$ is a strongly one-ended connected space in $\mathcal{P}$ then $p-\operatorname{Cat}(X) \leq p-\operatorname{cat}(X)+1$.

Proof. Let $\left\{U_{1}, \ldots, U_{k}\right\}$ be a family of p-categorical closed subsets with $X=U$ int $U_{i}$. If $\tilde{X}=\cup C_{p} U_{i}$ is the space in the proof of (2.5), we clearly have $p-\operatorname{Cat}(\tilde{X}) \leq k$. Moreover, the homotopy invariance of the push-out construction in any cofibration category ([5, II.1.2]) yields $\tilde{X} \simeq_{p} X \vee\left(\Sigma_{p} U_{1}\right) \vee \ldots \vee\left(\Sigma_{p} U_{k}\right)$ where " $\vee$ " denotes the wedge in $\mathcal{P}$. As $X$ is strongly one-ended we can assume that all the proper wedges are constructed along the same ray $\alpha: J \rightarrow X$. Now we get $X \simeq_{p} X \vee C_{p} Z$ where $Z=\Sigma_{p} U_{1} \vee \ldots \vee \Sigma_{p} U_{k}$, and so $p-\operatorname{Cat}(X) \leq k+1$ by (2.7).

Remark 2.10. If $K$ is the Berstein-Hilton space with $\operatorname{cat}(K)=2<3=\operatorname{Cat}(X)$ ([22]), it is clear that $X=K \times \mathbb{R}$ verifies $p-\operatorname{cat}(X)=4<6=p-\operatorname{Cat}(X)$. However, we do not know whether Proposition 2.9 is true for spaces with only one Freudenthal end.

We now apply (2.7) above to show (cf. ([22, 2.2])). 
Corollary 2.11. Let $X$ be a connected space in $\mathcal{P}$. Then $p-\operatorname{Cat}(X) \leq 2$ if and only if $X$ has the p-homotopy type of a proper suspension in $\mathcal{P}$. Moreover, if $X=$ int $P \cup$ int $Q$ with $P, Q$ p-homotopically trivial closed subspaces then $X \simeq_{p} \Sigma_{p}(P \cap Q)$.

Proof. According to the proof of (2.7) one gets that $X$ has the same p-homotopy type as the cofibre of the inclusion $i: P \cap Q \mapsto P$. Hence $X \simeq_{p} P /{ }^{p} P \cap Q \simeq_{p} C_{p} i$. As $P \simeq_{p} J$, it follows that $X \simeq_{p} \Sigma_{p}(P \cap Q)$.

As an immediate consequence of (2.5) and (2.11) we get

Corollary 2.12. If $X$ is a connected space in $\mathcal{P}$ with $p-\operatorname{cat}(X) \leq 2$ then $X$ is $a$ $p$-retract of a proper suspension. Furthermore, if $X=$ int $P \cup$ int $Q$ with $P$ and $Q$ p-deformable closed subspaces then $X \simeq_{p} \Sigma_{p}(P \cap Q)$.

\section{Proper category and the fundamental pro-group}

It is known that if a space has L-S category 2 then its fundamental group is free $([10,23.1])$. In this section we state some results concerning spaces with proper category 2. In particular we prove that the proper homotopy type of an open manifold with proper category 2 is $\mathbb{R}^{n}$. For this we recall the basic algebraic pro-invariants in proper homotopy theory.

Given a category $\mathcal{C}$, the category of towers of $\mathcal{C}$, tow $-\mathcal{C}$, is the category of inverse sequences $\underline{\mathbf{A}}=\left\{A_{1} \leftarrow A_{2} \leftarrow \ldots\right\}$ in $\mathcal{C}$ and pro-morphisms. See [23] for a complete reference about pro-categories. We are interested in the full subcategory of $\mathcal{M}$ or $($ tow $-\mathcal{C}$ ) whose objects are arrows $f: \underline{\mathbf{X}} \rightarrow A$ where $\underline{\mathbf{X}}$ is a (tow $-\mathcal{C}$ )-object and $A$ is a $\mathcal{C}$-object regarded as a constant tower whose bonding maps are the identity. This category is denoted $($ tow $-\mathcal{C}, \mathcal{C})$. A $($ tow $-\mathcal{C}, \mathcal{C})$-morphism from $f: \underline{\mathbf{X}} \rightarrow A$ to $g: \underline{\mathbf{Y}} \rightarrow B$ can be regarded as a $\mathcal{C}$-morphism between $A$ and $B$ and a (tow $-\mathcal{C}$ )morphism from $\underline{\mathbf{X}}$ to $\underline{\mathbf{Y}}$ such that both morphisms are compatible via the bonding maps. We shall use the above constructions for $\mathcal{C}=\mathcal{G} r, \mathcal{A} b$, the categories of groups and abelian groups respectively.

Given a ray $\alpha: J \rightarrow X$, the $n$-th homotopy pro-group of $(X, \alpha)$ is the inverse sequence in $($ tow $-\mathcal{G r}, \mathcal{G r})$

$$
\text { pro }-\pi_{n}(X, \alpha)=\left\{\pi_{n}\left(X, x_{0}\right) \leftarrow \pi_{n}\left(U_{1}, x_{1}\right) \leftarrow \pi_{n}\left(U_{2}, x_{2}\right) \leftarrow \ldots\right\}
$$

where $\left\{U_{j}\right\}$ is a system of $\infty$-neighbourhoods, $x_{j}=\alpha\left(t_{j}\right)$ with $\alpha\left(\left[t_{j}, \infty\right)\right) \subseteq U_{j}$, and the bonding morphisms are induced by inclusions and base-point change isomorphisms. When $n=1$ pro $-\pi_{1}(X, \alpha)$ will be called the fundamental pro-group of $(X, \alpha)$. It is known that the set of strong ends of $X, F(X)$ is in $1-1$ correspondence with the (pointed) set $\lim ^{1}$ pro $-\pi_{1}(X, \alpha)$ (see [21]).

The n-th homology pro-group of $X$ is defined as the inverse sequence in $($ tow $-\mathcal{A} b, \mathcal{A} b)$ 


$$
\text { pro }-H_{n}(X)=\left\{H_{n}(X) \leftarrow H_{n}\left(U_{1}\right) \leftarrow H_{n}\left(U_{2}\right) \leftarrow \ldots\right\} \text {. }
$$

Next we describe the fundamental pro-group of proper suspensions.

Lemma 3.1. If $\Sigma_{p} X$ is the proper suspension of $X$ in $\mathcal{P}$, pro $-\pi_{1}\left(\Sigma_{p} X, \alpha\right)$ is proisomorphic to an inverse sequence of free groups.

Proof. Let $r: X \rightarrow J$ be any p-map. We can use the $\infty$-neighbourhood system $\left\{U_{j}\right\}$ of $X$ with $U_{j}=r^{-1}([j, \infty))$. In this way, it is easily checked that $\left\{\Sigma_{p} U_{j}\right\}$ is a system of $\infty$-neighbourhoods of the proper suspension $\Sigma_{p} X$, where $\Sigma_{p} U_{j}$ is constructed by using $\left.r\right|_{U_{j}}: U_{j} \rightarrow[j, \infty)$. As the proper suspension has the same ordinary homotopy type as the ordinary suspension, one gets that pro $-\pi_{1}\left(\Sigma_{p} X, \alpha\right)$ is pro-isomorphic to the inverse sequence of free groups $\pi_{1}\left(\Sigma_{p} U_{j}, x_{j}\right)$.

Remark 3.2. An explicit description of the pro-group pro $-\pi_{1}\left(\Sigma_{p} X, \alpha\right)$ can be given as follows. With the notation of the proof of (3.1) we fix a component $c_{0}^{i} \in H_{0}\left(U_{i} ; \mathbb{Z}_{2}\right)$ for each $i$. Then each component $c^{i} \in H_{0}\left(U_{i} ; Z_{2}\right) c^{i} \neq c_{0}^{i}$, defines a generator $x\left(c_{i}, c_{i}^{0}\right)$ $\in \pi_{i}\left(\Sigma_{p} U_{i}, x_{j}\right)$ represented by a loop in $\Sigma_{p} U_{i}$ which only meets $U_{i}$ in two points, one in $c^{i}$ and the other in $c_{0}^{i}$. Then pro $-\pi_{1}\left(\Sigma_{p} X, \alpha\right)$ is pro-isomorphic to the inverse sequence

$$
\underline{\mathbf{S}}=\left\{\sqcup_{\left(c, c_{0}\right)} \mathbb{Z} \leftarrow \sqcup_{\left(c^{1}, c_{0}^{1}\right)} \mathbb{Z} \leftarrow \ldots\right\}
$$

where $\alpha: J \rightarrow \Sigma_{p} X$ is the canonical ray, "U" denotes free product and the bonding morphisms of $\underline{\mathbf{S}}$ carry $x\left(c^{i}, c_{0}^{i}\right)$ to $x\left(c^{i-1}, c_{0}^{i-1}\right)$ if $c^{i}+c_{0}^{i}=c^{i-1}+c_{0}^{i-1}$ in $H_{0}\left(U_{i-1} ; \mathbb{Z}_{2}\right)$.

Lemma 3.3. If $X$ is a properly admissible space in $\mathcal{P}$ and $\mathcal{B}(X)=\emptyset$ the bonding morphisms of $\underline{\mathbf{S}}$ are onto.

Proof. If $\left\{C_{j}\right\}_{j \geq 1}$ are the components, we can assume that $C_{j} \subseteq U_{j-1}$ and the result follows from the definition of $\underline{\mathbf{S}}$.

Lemma 3.4. If $X$ is a properly admissible space in $\mathcal{P}$ and $U(X)=\emptyset$, the bonding morphisms of $\underline{\mathbf{S}}$ are induced by basis inclusions.

Proof. Since $\mathcal{U}(X)=\emptyset$ all the components $\left\{C_{j}\right\}_{j \geq 1}$ of $X$ are compact, and we can choose $U_{i}=\cup_{j \geq i} C_{j}$. Now the result follows easily from the definition of $\underline{\mathbf{S}}$.

Corollary 3.5. If $X$ is a connected space in $\mathcal{P}$ with $p-\operatorname{cat}(X) \leq 2$ then pro $-\pi_{1}(X)$ is pro-isomorphic to an inverse sequence of free groups.

Proof. The inverse sequence pro $-\pi_{1}(X, \alpha)$ is pro-isomorphic to its image in pro $-\pi_{1}\left(\Sigma_{p} Y, \alpha\right)$ for some $Y$ in $\mathcal{P}$ according to (2.12). Now the result follows from (3.1). 
Corollary 3.6. Let $X$ be a one-ended connected space in $\mathcal{P}$ such that $X=$ int $P \cup$ int $Q$ with $P, Q$ properly admissible closed subspaces $p$-deformable to $J$ in $X$. Assume that $\mathcal{B}(P \cap Q)=\emptyset$. Then $X$ is strongly one-ended.

Proof. According to (3.3) all the bonding morphisms of the inverse sequence pro $-\pi_{1}\left(\Sigma_{p}(P \cap Q), \alpha\right)$ are onto, and so $\lim _{\leftarrow}^{1}$ pro $-\pi_{1}\left(\Sigma_{p}(P \cap Q), \alpha\right)=\{[\alpha]\}$ (see ([19, II.6.2])). Hence $\Sigma_{p}(P \cap Q)$ has only one strong end. Finally, by (2.12) we know that there exists a p-retraction $r: Z \rightarrow X$ where $Z \simeq_{p} \Sigma_{p}(P \cap Q)$. This retraction implies that $X$ is strongly one-ended.

Corollary 3.7. Let $X$ be an one-ended metrizable $A N R$ in $\mathcal{P}_{0}$ such that pro- $H_{1}(X)$ is trivial in (tow $-\mathcal{A} b, \mathcal{A} b)$. Assume that $p-\operatorname{cat}(X)=2$. Then pro $-\pi_{1}(X, \alpha)$ is trivial in $($ tow $-\mathcal{G r}, \mathcal{G r}$ ).

Recall that a space $X$ is $1-L C$ at $\infty$ if for any $\infty$-neighbourhood $U \subseteq X$ there exists an $\infty$-neighbourhood $V \subseteq U$ such that any loop in $V$ is homotopically trivial in $U$. Hence the triviality of pro $-\pi_{1}(X, \alpha)$ is equivalent to say that $X$ is simply connected and $1-\mathrm{LC}$ at $\infty$.

Proof. Let $P, Q$ be two properly admissible closed subspaces p-deformable to $J$ with $X=$ int $P \cup$ int $Q$ (see (2.2)). If $\mathcal{U}(P)$ or $\mathcal{U}(Q)$ is empty then $\mathcal{U}(P \cap Q)=\emptyset$. Otherwise we can apply (2.3) and (2.4) to assume that $\mathcal{B}(P \cap Q)=\emptyset$. Now the result follows from (2.12) and (3.2) and the following result.

Proposition 3.8. Let $\underline{\mathrm{A}}=\left\{A_{0} \leftarrow A_{1} \ldots \leftarrow \ldots\right\}$ be an inverse sequence of groups which is a retract in (tow $-\mathcal{G} r, \bar{G} r)$ of one of the following inverse sequences

$$
\begin{aligned}
& \text { (a) } \underline{\mathbf{L}}=\left\{L_{0} \stackrel{k_{1}}{\leftarrow} L_{1} \stackrel{k_{2}}{\leftarrow} L_{2} \ldots\right\} \\
& \text { (b) } \underline{\mathbf{P}}=\left\{P_{0} \stackrel{p_{1}}{\leftarrow} P_{2} \stackrel{p_{2}}{\leftarrow} P_{2} \ldots\right\}
\end{aligned}
$$

where $P_{i}$ and $L_{i}$ are free groups, $k_{i}$ are basis inclusions, and $p_{i}$ are epimorphisms. Then $\underline{\mathbf{A}}$ is trivial in (tow $-\mathcal{G} r, \mathcal{G}$ r) if and only if its abelianization $\underline{\mathbf{A}}^{a b}=\left\{A_{0}^{a b} \leftarrow A_{1}^{a b} \ldots \leftarrow \ldots\right\}$ is trivial in (tow $-\mathcal{A} b, \mathcal{A} b)$.

In the proof of (3.8) we shall use Lemma 3.9 below on commutator subgroups. Given a group $G$, the subgroup $[G, G]^{n}(n \geq 1)$ is defined inductively as $[G, G]^{1}=[G, G]$ (the commutator subgroup of $G$ ) and $[G, G]^{n}=\left[[G, G]^{n-1},[G, G]^{n-1}\right]$. Moreover if $F$ is a free group the subgroup $G \subseteq F$ is called a free factor if a basis of $G$ can be extended to a basis of the whole of $F$.

Lemma 3.9. Let $G \hookrightarrow F$ a free factor. Then $[G, G]^{n}$ is a free factor of $[F, F]^{n}$ and $[F, F]^{n} \cap G=[G, G]^{n}$ for all $i \geq 1$. 
Proof. The commutator $[F, F]$ consists of all words $w \in F$ such that the sum of coefficients of each generator in $w$ is trivial. This directly implies $[G, G]=[F, F] \cap G$ since $G$ is a free factor. In particular, this equality yields $[F, F]^{2} \cap G=[F, F]^{2} \cap[G, G]$. Now we apply $([18$, Ex. 32, p. 117]) to $[F, F] \subseteq F$ to show that $[G, G]$ is a free factor in $[F, F]$, and then $[G, G]^{2}=[F, F]^{2} \cap[G, G]=[F, F]^{2} \cap G$ by the same result. We can now proceed inductively.

Proof of (3.8). Assume $\underline{\mathbf{A}}^{a b}$ is trivial in $($ tow $-\mathcal{A} b, \mathcal{A} b)$. If $\underline{\mathbf{A}} \stackrel{\mathbf{k}}{\rightarrow} \underline{\mathbf{P}} \stackrel{\mathbf{r}}{\rightarrow} \underline{\mathbf{A}}$ is a retraction in $($ tow $-\mathcal{G} r, \mathcal{G} r)$, then $\mathbf{k}$ is a monomorphism in $($ tow $-\mathcal{G} r, \mathcal{G} r)$ and there exists a pro-isomorhism $\underline{\mathbf{A}} \stackrel{\phi}{\cong} \underline{\mathbf{B}}=\left\{B_{i}\right\}$ with $B_{i}=\operatorname{Im} k_{i}$ for a representative $\left\{k_{i}: A_{m_{1}} \rightarrow P_{i}\right\}$ of k. Hence we have a retract $\underline{\mathbf{B}} \stackrel{\mathbf{k}^{\prime}}{\rightarrow} \underline{\mathbf{P}} \stackrel{\mathfrak{r}^{\prime}}{\rightarrow} \underline{\mathbf{B}}$ by using $\phi$.

On the other hand $\underline{\mathbf{B}}$ is also pro-isomorphic to the inverse sequence $\underline{\mathbf{C}}=\left\{C_{i}\right\}$ with $C_{i}=I m r_{i}^{\prime} \subseteq B_{i}$ for a representative $\left\{r_{i}^{\prime}: P_{n_{i}} \rightarrow B_{i}\right\}$ of $\mathbf{r}^{\prime}$. In particular, $\underline{\mathbf{C}}^{a b}=0$. Moreover, since the bonding morphisms of $\underline{\mathbf{P}}$ are onto, it follows that the bonding morphisms of $\underline{\mathbf{C}}$ are also onto. As the abelianization functor preserves epimorphisms, it follows $C_{i}^{a b}=\overline{0}$ for all $i$, and hence $C_{i}=0$ since $C_{i} \subseteq B_{i}$ is a free group. Therefore $\underline{\mathbf{A}}=0$.

Assume now that $\underline{\mathbf{A}} \stackrel{\mathbf{L}}{\rightarrow} \underline{\mathbf{L}} \stackrel{r}{\rightarrow} \underline{\mathbf{A}}$ is a retraction in $($ tow $-\mathcal{G} r, \mathcal{G r})$. As above we can replace this retract by a new retract $\underline{\mathbf{B}} \stackrel{\mathbf{k}^{\prime}}{\rightarrow} \underline{\mathbf{L}} \stackrel{\stackrel{r}{\prime}^{\prime}}{\rightarrow} \underline{\mathbf{B}}$ where $\underline{\mathbf{B}}$ is an inverse sequence of free groups. Moreover, as the bonding morphisms of $\underline{\mathbf{L}}$ are injective the bonding morphisms of $\underline{\mathbf{B}}$ are also injective. More explicitly, we have an increasing sequence of positive integers $n_{1}<n_{2}<\ldots$ and commutative diagrams

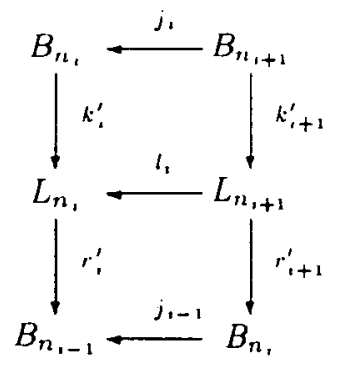

where all morphisms are injective except the $r_{i}$ 's, and $r_{i+1}^{\prime} k_{i+1}^{\prime}=j_{i}$ for all $i$.

As $\underline{\mathbf{A}}^{a b}=\underline{\mathbf{B}}^{a b}=0$ we can assume without loss of generality that $j_{i}^{a b}=0$ and hence $B_{n_{i+1}}$ is contained in the commutator subgroup $\left[B_{n_{i}}, B_{n_{i}}\right] \subseteq\left[L_{n_{i}}, L_{n_{i}}\right]$. Therefore $B_{n_{i+2}} \subseteq L_{n_{i+2}} \cap\left[L_{n_{i}}, L_{n_{i}}\right]^{2}$, and so $B_{n_{i+2}} \subseteq\left[L_{n_{i+2}}, L_{n_{i+2}}\right]^{2}$ by (3.9). In fact the same argument shows $B_{n_{j}} \subseteq\left[L_{n_{i}}, L_{n_{i}}\right]^{2}$ for all $i \geq 3$. Hence $B_{n_{i+2}} \subseteq\left[B_{n_{i+1}}, B_{n_{i+1}}\right] \subseteq\left[L_{n_{i+1}}, L_{n_{i+1}}\right]^{3} \subseteq\left[L_{n_{i}}, L_{n_{i}}\right]^{3}$. Then (3.9) yields $B_{n_{i+2}} \subseteq\left[L_{n_{i+2}}, L_{n_{i+2}}\right]^{3}$; that is, the inclusion $B_{n_{i}} \subseteq\left[L_{n_{i}}, L_{n_{i}}\right]^{3}$ holds for all $i \geq 3$ and we now apply inductively (3.9) to get all $i \geq 3 B_{n_{i}} \subseteq \cap_{m \geq 1}\left[L_{n_{i}}, L_{n_{i}}\right]^{m}=0$ (see $[17$, I.3.4]). Therefore $\underline{\mathbf{A}} \cong \underline{\mathbf{B}}=0$ in $($ tow $-\mathcal{G r}, \mathcal{G} r)$. 
We devote the rest of the paper to characterize the manifolds whose proper L-S category is 2 . The proof of the next result is part of the proof of $([2,3.6])$.

Proposition 3.10. If $M$ is a connected open $n$-manifold with $p-\operatorname{cat}(M)=2$ then $M$ is homologically trivial and simply connected. In particular $M$ is contractible.

We briefly recall the proof. As $\operatorname{cat}(M) \leq p-\operatorname{cat}(M)=2$ the fundamental group $\pi_{1}(M)$ is free by $([10,23.1])$. Also $p-\operatorname{cat}(M)=2$ implies that cup - length $(M)=1$ for the cup-product $H_{c}^{*}\left(M ; \mathbb{Z}_{p}\right) \oplus H^{*}\left(M ; \mathbb{Z}_{p}\right) \rightarrow H_{c}^{*}\left(M ; \mathbb{Z}_{p}\right)$ for all prime $p$. Here $H_{c}^{*}$ is the cohomolgy of compact supports. This fact together with the Poincaré Duality yield that $M$ is homologically trivial and as $H_{1}(M)=\operatorname{abelianized}\left(\pi_{1}(M)\right)$ is free with the same rank as $\pi_{1}(M)$ it follows that $M$ is also simply connected. The result follows from the Whitehead Theorem ([26, IV.7.13]).

The following result gives us the pro-homology at $\infty$ of homologically trivial manifolds.

Proposition 3.11. Any homologically trivial connected open $n$ manifold is one-ended and pro $-H_{q}(M)=0$ if $q \neq 0, n-1$ and pro $-H_{p}(M)$ is pro-isomorphic to the constant inverse sequence $\{\mathbb{Z}=\mathbb{Z}=\ldots\}$ for $p=0, n-1$.

Proof. The manifold $M$ is one-ended by $\left(\left[13\right.\right.$, I.7]). Let $H_{*}^{\infty}$ and $H_{*}^{e}$ denote the homology of infinite cycles and the homology at infinity respectively. Then the exact sequence $([\mathbf{2 0}, \mathrm{p} .306])$

$$
\ldots \rightarrow H_{q}(M) \rightarrow H_{q}^{\infty}(M) \rightarrow H_{q}^{e}(M) \rightarrow H_{q-1}(M) \rightarrow \ldots
$$

yields isomorphisms $H_{q}^{\infty}(M) \cong H_{q}^{e}(M)$ for $q \geq 2$ and $H_{1}^{e}(M) \cong H_{0}(M) \cong \mathbb{Z}$ since $H_{0}^{\infty}(M)=0$. Now the Poincaré Duality $H_{q}^{\infty}(M) \cong H^{n-q}(M)$ yields that $H_{q}^{\infty}(M)=0$ if $q \neq n$. If $\left\{W_{j}\right\}$ denotes a system of $\infty$-neighbourhoods of $M$ we have the Milnor exact sequence $([3,2.1])$.

$$
0 \rightarrow \lim _{\leftarrow}^{\prime}\left\{H_{q+1}\left(W_{j}\right) \rightarrow H_{q}^{e}(M) \rightarrow \lim _{\leftarrow}\left\{H_{q}\left(W_{j}\right)\right\} \rightarrow 0\right.
$$

and the groups $\lim ^{1}\left\{H_{p}\left(W_{j}\right)\right\}$ and $\lim \left\{H_{q}\left(W_{j}\right)\right\}$ are trivial if $p \geq 0$ and $q \neq 0, n-1$ and $\lim _{\leftarrow}\left\{H_{q}\left(W_{j}\right)\right\} \cong \mathbb{Z}$ if $q=0, n-1$. Hence the inverse sequence $\left\{H_{q}\left(W_{j}\right)\right\}$ is trivial if $q \neq 0$, $n-1$ by $([19,6.2$ Lemma 2$])$ and $\left\{H_{q}\left(W_{j}\right)\right\}$ is pro-isomorphic to the constant sequence $\{\mathbb{Z}=\mathbb{Z}=\ldots\}$ if $q=0, n-1$ by $([19,6.2$ Corollary 8$])$.

As an immediate consequence of (3.7), (3.10), and (3.11) one gets

Corollary 3.12. Any connected open n-manifold $M(n \geq 3)$ with $p-\operatorname{cat}(M)=2$ is $1-L C$ at $\infty$. 
As a consequence of (3.10) and (3.12), the homotopical characterizations of Euclidean spaces due to Siebenmann ([23]) and Freedman ([11]) can be restated in the following way

Theorem 3.13. (cf. $[2,3.6])$ A connected open n-manifold $M(n \neq 3)$ is homeomorphic to $\mathbb{R}^{n}$ if and only if $p-\operatorname{cat}(M)=2$.

Remark 3.14. The characterization in dimension 3 depends on the Poincare Conjecture and (3.13) can be only stated for irreducible open 3-manifolds as a consequence of [25]. We recall that a 3-manifold is irreducible if every embedded 2sphere bounds a 3-cell.

For any dimension we can use basic results of proper homotopy to show

Theorem 3.15. (cf. $[15$, p. 336]) Let $M$ be a connected (triangulated) open $n$ manifold. Then $p-\operatorname{cat}(M)=2$ if and only if $M$ has the same proper homotopy type as $\mathbb{R}^{n}$.

Proof. Assume $p-\operatorname{cat}(M)=2$. Then $M$ is contractible by $(3.10)$ and so $M=\mathbb{R}^{n}$ for $n \leq 2$. If $n \geq 3$ we know (see the proof of (3.11)) that $H_{q}^{\infty}(M)=0$ if $q \neq n$. Since pro $-\pi_{1}(M)=0$ by (3.7), the proper Hurewicz Theorem for CW-complexes in $([3,3.4])$ yields $H_{n}^{\infty}(M) \cong\left[\mathbb{R}^{n}, M\right]_{p}$, and the fundamental class $\mu_{M} \in H^{\infty}(M)$ can be represented by a p-map $f: \mathbb{R}^{n} \rightarrow M$. Moreover $f_{*}: H_{n}^{\infty}\left(\mathbb{R}^{n}\right) \rightarrow H_{n}^{\infty}(M)$ is an isomorphism and by ([3, 3.8]) we conclude that $f$ is a p-homotopy equivalence.

Remark 3.16. Since any open $n$-manifold has the p-homotopy type of a finite dimensional locally finite polyhedron ([16, p. 123]), Theorem (3.15) actually holds for any topological manifold.

Acknowledgement. This work was partially supported by the project DGICYT PB92-0672.

\section{REFERENCES}

1. R. Ayala, E. Domínguez and A. Quintero, A theoretical framework for proper homotopy theory, Math. Proc. Cambridge Philos. Soc. 107 (1990), 475-482.

2. R. Ayala, E. Dominguez, A. Márquez and A. Quintero, Lusternik-Schnirelmann invariants in proper homotopy, Pacific J. Math. 153 (1992), 201-215.

3. R. Ayala, E. Dominguez and A. Quintero, Hurewicz Theorem for homology at infinity, Quart. J. Math. Oxford (2) 44 (1993), 139-153.

4. B. J. Ball and R. B. Sher. A theory of proper shape for locally compact metric spaces, Fund. Math. 86 (1974), 162-192.

5. H. J. BaUEs, Algebraic Homotopy (Cambridge Studies in Advanced Maths., 15, Cambridge Univ. Press, 1989). 
6. M. ClapP and D. PuPPE, Invariants of the Lusternik-Schnirelmann type and the topology of critical sets, Trans. Amer. Math. Soc. 298 (1986), 603-620.

7. O. CoRneA, Cone-length and Lusternik-Schnirelmann category, Topology 33 (1993), 95-111.

8. D. A. EDWARDS and H. M. HASTINGS, Čech and Steenrod homotopy theories with applications to Geometric Topology (Lecture Notes 542, Springer, 1976).

9. R. Engelking and K. Sieklucki, Topology. A geometric approach (Heldermann, 1992).

10. R. H. Fox, On the Lusternik-Schnirelmann category, Ann. of Math. 42 (1941), 333-370. 453.

11. M. H. Freedman, The topology of 4-manifolds, J. Differential Geom. 17 (1982), 375-

12. T. GANEA, Lusternik-Schnirelmann category and strong category, Illinois J. Math. 11 (1967), 417-427.

13. L. Glaser, Geometrical Combinatorial Topology. Vol II (Van Nostrand, 1972).

14. K. Hess and J. M. Lemaire, Generalizing a definition of Lusternik and Schnirelmann to model categories, J. Pure Appl. Algebra 91 (1994), 165-182.

15. I. M. JAMES, On category in the sense of Lusternik-Schnirelmann, Topology 17 (1978), 331-349.

16. R. C. Kirby and L. SiebenmanN, Foundational essays on topological manifolds, smoothings, and triangulations (Annals of Math. Studies, 88, Princeton Univ. Press, 1977).

17. R. C. Lyndon and P. E. Schupp, Combinatorial Group Theory (Springer, 1977).

18. W. Magnus, A. Karrass and D. Solitar, Combinatorial Group Theory (Wiley, 1966).

19. S. MARdeŠIĆ and J. SEgal, Shape Theory (North-Holland, 1982).

20. W. S. MASSEY, Homology and Cohomology Theory (M. Dekker, 1978).

21. M. Mihalik, Semistability at the end of a group extension, Trans. Amer. Math. Soc. (1983), 307-321.

22. L. Montejano, Lusternik-Schnirelmann category: A geometric approach (Banach Center Publications 18. PWN, 1986), 119-131.

23. L. SiebenmanN, On detecting Euclidean Space homotopically among topological manifolds, Invent. Math. 6 (1968), 245-261.

24. F. TAKENS, The Lusternik-Schnirelmann categories of a product space, Compositio Math. $22(1970), 175-180$.

25. C. T. C. WALL, Open 3-manifolds which are 1-connected at infinity, Quart. J. Math. Oxford (2) 16 (1965), 263-268.

26. G. W. WhiteHEAd, Elements of Homotopy Theory (GTM, 61. Springer, 1978).

Departamento de Geometria y Topología

Facultad de Matemáticas

UNIVERSIDAD de SEVILla

APARTADO 1160

41080-SEVILLA

SpaIN 